\title{
Neural Correlates of Humor Detection and Appreciation in Children
}

\author{
Michelle N. Neely, ${ }^{1}$ Elizabeth Walter, ${ }^{1}$ Jessica M. Black, ${ }^{1,2}$ and Allan L. Reiss ${ }^{1,3}$ \\ ${ }^{1}$ Center for Interdisciplinary Brain Sciences Research, Stanford University, Stanford, California 94305, ${ }^{2}$ Graduate School of Social Work, Boston College, \\ Chestnut Hill, Massachusetts 02467, and 3Departments of Psychiatry and Radiology, Standard University, Stanford, California 94305
}

Humor is a vital component of human well-being. Neuroimaging studies conducted with adults indicate that humor activates specific brain regions, including the temporo-occipito-parietal junction (TOPJ), involved in incongruity resolution, and mesolimbic regions, involved in reward processing. However, no study to date has used neuroimaging to examine humor in typically developing children. Here, we illuminate the neural network involved in the detection and appreciation of humor in childhood.

Fifteen typically developing children (ages, 6-12 years) were invited to watch and respond to video clips while neural activity was imaged with a 3T GE Discovery MR750 scanner. Before presentation during functional imaging, the clips were evaluated by age-matched controls and were representative of three categories: Funny, Positive (enjoyable but not funny), and Neutral (not intended to evoke any emotional response). We found TOPJ and mesolimbic activation in children's response to humor, suggesting these regions may form a humor-essential neural network already present in childhood. Furthermore, in a novel comparison of Funny to Positive stimuli, we found that bilateral TOPJ activation may be specific to humor processing and not part of a general constellation of neural activity in response to reward. Finally, we observed greater activation in the inferior frontal gyrus and nucleus accumbens in younger participants, indicating humor activation intensity changes during development.

By providing a crucial link in studying the neurodevelopment of humor processing across the lifespan, our findings contribute valuable information about the evolution of how children understand their world.

\section{Introduction}

While some might consider humor's playful role trivial, extant findings point to its critical role in building and maintaining relationships, emotional health, and cognitive function (Martin, 2007). Because of humor's myriad psychological and biological effects, an improved understanding of the neural systems involved in its development is of particular relevance to studying human health and behavior.

Previous research has implicated brain regions associated with the detection of incongruity in the interpretation of humor. These regions include the inferior temporal gyrus, the left fusiform gyrus, and other areas within the overlapping cortical regions between the left temporal, occipital and parietal lobes: the temporal-occipital-parietal junction (TOPJ), which includes Brodmann areas 37, 39, and 40 (Mobbs et al., 2003; Samson et al., 2009). Humor processing in adults has also been shown to acti-

Received Aug. 12, 2011; revised 0ct. 28, 2011; accepted Dec. 6, 2011.

Author contributions: M.N.N., E.W., J.M.B., and A.L.R. designed research; M.N.N. and E.W. performed research; M.N.N., E.W., J.M.B., and A.L.R. analyzed data; M.N.N., E.W., J.M.B., and A.L.R. wrote the paper.

This work was supported by grants from the Lucile Packard Foundation for Children's Health, Spectrum Child Health, Clinical and Translational Science Award to A.L.R. and J.M.B., and the Bette and Al Moorman Young Investigator Award to A.L.R. Funding for J.M.B. was provided by an NIH-sponsored institutional research training grant (T32) 5T32MH019908-17 to A.L.R. Funding for M.N.N. was provided through a grant from the Vice Provost for Undergraduate Education at Stanford University. We thank John Lyman, Emilie Qiao, and Jennifer Gonzales for support in behavioral data collection.

Correspondence should be addressed to Dr. Allan Reiss, Department of Psychiatry and Behavioral Sciences, 401 Quarry Road, Stanford, CA 94305-5795. E-mail: areiss1@stanford.edu.

DOI:10.1523/JNEUROSCI.4172-11.2012

Copyright $\odot 2012$ the authors $\quad 0270-6474 / 12 / 321784-07 \$ 15.00 / 0$ vate mesolimbic reward circuitry, including the nucleus accumbens (NAcc) (Mobbs et al., 2003). These structures, implicated in the detection and appreciation of reward (Schultz, 2002), are hypothesized to be responsible for the pleasurable nature of humor comprehension (Mobbs et al., 2003).

Humor-related research in children has been confined to the behavioral realm, where the development of humor has been linked to children's growing cognitive mastery and social competence (Zigler et al., 1966; Bainum et al., 1984). The development of humor parallels children's growing cognitive and perceptual skills. Humorous stimuli too deviant from a child's developmental stage - whether too simplistic or too complex-are not perceived to be as pleasurable (Pinderhughes and Zigler, 1985; Johnson, 1997). Deficits in humor comprehension may signal the presence of learning disabilities (Bruno et al., 1987). Because humor is closely related to empathy and intuition about another's intent, humor detection/appreciation is often affected in pathologies where a child's social functioning is impaired (St. James and Tager-Flusberg, 1994).

To our knowledge, no study has used neuroimaging to examine humor in typically developing children-a surprising gap in the literature, given humor's interrelation with the development of cognitive and social competence. In this study, we sought to illuminate the cortical and subcortical regions responsible for humor processing, involving detection and resolution of incongruity as well as the appreciation of reward, in children ages 6-12 years old.

Based on the previous literature in adults, we hypothesized that the TOPJ and NAcc would be activated by Funny stimuli as 
compared with Neutral stimuli. By contrasting activation due to the processing of Funny stimuli to activation observed in response to Positive (but non-funny) stimuli, we were able to distinguish neural activation that is essential to humor processing specifically. We predicted that the TOPJ would remain active in this contrast, while the NAcc and mesolimbic reward processing would show no difference using this subtraction methodology.

The elucidation of a humor-essential neural network in children could provide valuable insight into how the neurological underpinnings of humor processing evolve over the lifespan. A better appreciation for how humor-specific neural networks function in children will improve our understanding of humor's longitudinal role in human health and behavior.

\section{Materials and Methods}

Participants. Fifteen typically developing children [9 female; mean age (SD): 10.0 (1.18) years; range, 7.9-11.7 years] were invited to complete cognitive and behavioral tests, watch video clip stimuli in a functional magnetic resonance imaging (fMRI) scanner, and provide ratings of how humorous and enjoyable they found each clip. All participants were right-handed, had no clinical psychiatric symptoms or problems as indicated by parental responses to medical history-related questions during a phone screening, and were of average to gifted intelligence [as assessed by the Wechsler Abbreviated Scale of Intelligence (WASI)]. Participants and parents provided assent and consent, respectively, before participation. The Stanford University Panel on Human Subjects in Medical Research approved the study.

Participants underwent two fMRI scans (each $\sim 15$ min in duration). Movement during scans was calculated based on the displacement and rotation required to realign functional scans at each time point with respect to a reference image. Participants with displaced scans that exceeded $21 \%$ of the total number of scans were excluded from further analysis. Of an initial scanned subject pool of 20 participants, three participants (all male) were excluded from analysis due to excess movement and one participant (female) was excluded from analysis for not completing the second segment of the in-scanner protocol. An additional male was removed from analysis because his brain mask did not include the temporal lobe (indicating that he moved significantly between the localizer scan and the functional scans). This resulted in an analyzed population of 15 participants.

Stimuli selection. Because our participants included children who differed in reading ability, we wanted to choose stimuli that did not depend solely on verbal humor. Instead, we explored the dynamic domain of humorous content by using video stimuli depicting funny and nonfunny scenes and events. To ensure that the videos we chose would be funny (or not funny) to our child participants, we pilot tested videos with a matched sample of children (who did not participate in scanning). Children, similar in demographic measures (such as age) to the scanned participants, watched a total of 455 videos (over two pilot viewing sessions). Participants rated these videos according to how funny they found the video (on a scale from $1=$ least funny to $8=$ most funny) and also rated how enjoyable they found the video [on a scale from $1=$ least enjoyable to $8=$ most enjoyable). Using mean ratings from both scales, we divided videos into three categories: Funny, Positive, and Neutral. Funny video clips were defined as having mean ratings $\geq 5$ on the funny scale; Neutral video clips were defined as having mean ratings $\leq 4$ on both the funny and enjoyable scales; and Positive video clips were defined as having mean ratings $\geq 5$ on the enjoyable scale but $\leq 4$ on the funny scale. It is worth noting that Funny video clips also received high mean ratings on the enjoyable scale, even though a minimum rating was not requisite for their categorization as funny. Intuitively this makes sense, as humor is intrinsically rewarding - this is why individuals actively seek, and are willing to pay money for, exposure to humorous content.

Representative clips were then coded along a number of dimensions, including video clip length, number of humans present, number of animals, presence/absence of talking, and presence/absence of music (see Table 4). Videos from each of the three categories were chosen so as to best match across these qualitative dimensions, while ensuring that Funny and Positive videos were equal for the enjoyable rating; that Funny videos had greater funny ratings than both Positive and Neutral; and that Neutral videos had enjoyable and funny ratings significantly lower than Funny clips, and had enjoyable ratings significantly lower than Positive videos.

Funny videos included scenes of people stumbling while skiing or running, a kid "swimming" on the floor, cars accidentally running into things, animals performing amusing tricks, a kid being catapulted into the air from an inflatable couch, and many others. Rewarding videos included scenes of dirt biking, gymnastics, dancing, snowboarding, a person jumping over a car, kids breakdancing, a performer playing music using water glasses, and many others. Neutral videos included kids riding bicycles, kids singing in school plays, a child meeting Mickey Mouse, nature documentary style videos of animals, and many others.

Experimental task. The in-scanner task comprised a set of 96 short color video clips previously viewed and evaluated by age-matched controls to represent three categories: Funny, Positive, and Neutral. Each stimulus category included 32 video clips, 16 of which were presented in the first functional run, with the remainder presented in the second functional run. Funny clips were rated as funny (and enjoyable), Positive clips were rated as enjoyable but non-funny, and Neutral clips were not intended to evoke any emotional response. Video clips were $\sim 12 \mathrm{~s}$ each. The order of video clips was randomized within trials, but not between participants. Stimuli were presented at the center of a viewing screen above the head coil using a custom-built MRcompatible projection system and EPrime 2.0 software (http://www.pstnet. com/eprime.cfm). Participants lying in the scanner viewed stimuli via an angled mirror. The software program Optseq (http://surfer.nmr.mgh. harvard.edu/optseq/) was used to optimally randomize the order and spacing between stimuli to ensure orthogonality of our stimulus conditions. Stimuli were divided into two runs so that participants would get a short break during the lengthy scan session. During the two runs $(\sim 30$ min total scan acquisition time), participants viewed all video clips and pressed a "Yes" button with their right index finger when they liked watching a video and a "No" button with their right middle finger when they did not like watching a clip. This simple in-scanner task was used to ensure that participants were attending to the stimuli.

Briefly, each trial started with a fixation cross that was presented for 200-13,000 ms (timing was based on Optseq output), after which the video started and played for its full duration (4000-16,000 ms). After the video played, the participant had $3000 \mathrm{~ms}$ to respond. Post-response, the screen was replaced with a fixation cross on black background until the onset of the next trial (Fig. 1).

Participants were familiarized with the task before undergoing imaging by playing a short $(\sim 2 \mathrm{~min}$ duration $)$ practice version of the task. Participants practiced in an MRI simulator before imaging and repracticed the task outside the scanner immediately before imaging. Stimuli used for the practice versions were different from those used in-scanner and featured different actors, activities, and scenes. Participants were encouraged to be honest in their assessments and were told that they were not expected to like all of the clips.

Post-imaging, participants were asked to rewatch the stimuli and to provide more nuanced ratings of the clips. Participants used an ordinal interval scale to indicate how funny they found the video (on a scale from $1=$ least funny to $8=$ most funny) and also to rate how enjoyable they found the video (on a scale from $1=$ least enjoyable to $8=$ most enjoyable). This aided the interpretation and validation of dichotomous inscanner ratings (Mobbs et al., 2003).

fMRI acquisition. Participants were scanned on a 3 tesla (GE Discovery MR750 scanner) MRI at Stanford's Lucas Center for Neuroimaging using a custom-built single-channel birdcage head coil optimized for fMRI scans. Over two runs, a total of 914 whole-brain volumes were collected on 30 axial-oblique slices ( $4.0 \mathrm{~mm}$ thick, $1.0 \mathrm{~mm}$ skip) prescribed parallel to the intercommissural (AC-PC) line, using a $\mathrm{T} 2{ }^{\star}$-weighted gradient echo spiral pulse sequence (Glover and Lai, 1998) sensitive to BOLD contrast with the following acquisition parameters: $\mathrm{TE}=30.0 \mathrm{~ms}, \mathrm{TR}=$ $2000 \mathrm{~ms}$, flip angle $=80^{\circ}, \mathrm{FOV}=22.0 \mathrm{~cm}$, number of slices $=31$, slice thickness $=4.0 \mathrm{~mm}$, acquisition matrix $=64 \times 64$, approximate voxel size $=3.4375 \times 3.4375 \times 4.0 \mathrm{~mm}$. An automated high-order shimming method based on spiral acquisitions was used before acquisition of fMRI scans to reduce blurring and signal loss arising from field inhomogeneities (Kim et al., 2002). A high-resolution T1-weighted three- 


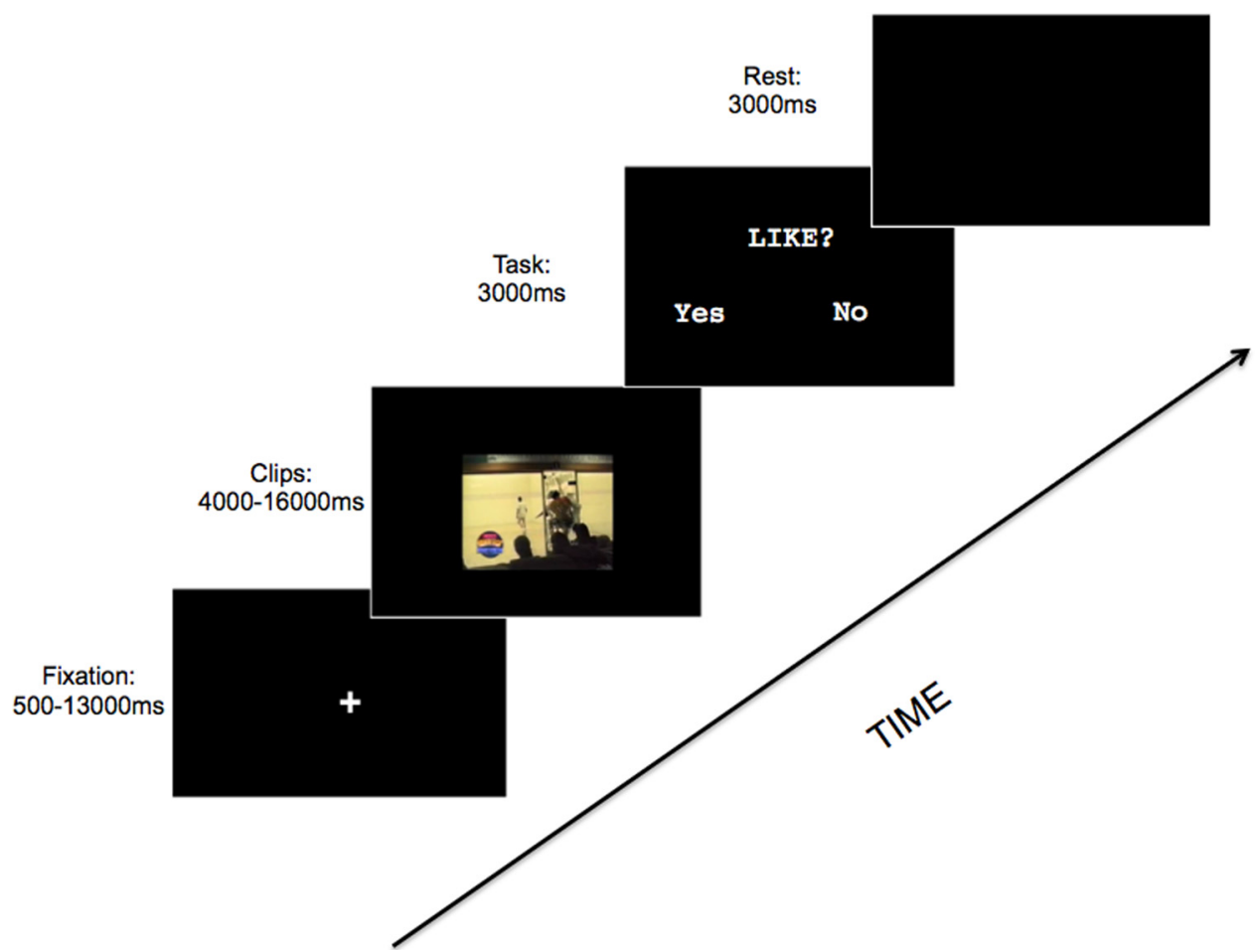

Figure 1. In-scanner experimental trial timeline. Participants viewed video clips (4000 - $16,000 \mathrm{~ms}$ in length) and were given $3000 \mathrm{~ms}$ following the clip to indicate with a button press whether $\mathrm{s} / \mathrm{he}$ liked (index finger button press) or did not like (middle finger button press) the video clip. Intertrial interval ranged from 200 to $13,000 \mathrm{~ms}$.

dimensional inversion recovery spoiled gradient-recalled acquisition was acquired for coregistration with the following parameters: $\mathrm{TE}=$ minimum, $\mathrm{TR}=8.1 \mathrm{~s}$, flip angle $=15^{\circ}, \mathrm{FOV}=224 \mathrm{~cm}$, slice thickness $=1.6$ $\mathrm{mm}, 124$ slices in the coronal plane; matrix $=256 \times 192$. The images were reconstructed as a $256 \times 256 \times 124$ matrix.

fMRI analysis. Statistical analyses were performed using Statistical Parametric Mapping software (SPM8; Wellcome Department of Cognitive Neurology, London, UK, http://www.fil.ion.ucl.ac.uk/spm/software/ spm8/). Each subject's data were slice-time corrected, realigned, resliced, spatially smoothed (4 mm FWHM), motion corrected, coregistered to the individual's anatomical image, and normalized to MNI space. In addition, we repaired movement and signal artifacts in our images using ArtRepairsoftware (http://cibsr.stanford.edu/tools/human-brain-project/ artrepair-software.html; Mazaika et al., 2009), developed in house at the Center for Interdisciplinary Brain Sciences Research and available for public download.

Statistical analysis. After preprocessing, each individual subject was analyzed using the general linear model (Friston et al., 1995) implemented in SPM8. Individual subject data were high-pass filtered at $120 \mathrm{~s}$, globally scaled, and analyzed using a fixed-effects model comprising the three separate experimental conditions (the rest were modeled implicitly). Group data were smoothed (7 mm FWHM) and the individual subject contrast images were entered into a random-effects analysis. SPM images were overlaid on a high-resolution canonical T1-weighted image for viewing. Coordinates of activation were converted from MNI to Talairach space using the mni2tal function (http://www.mrc-cbu.cam.ac. $\mathrm{uk} / \mathrm{Imaging} / \mathrm{Common} / \mathrm{mnispace} . \mathrm{shtml}$ ). Brain regions were then identified from these $x, y$, and $z$ coordinates using the Talairach Daemon (Research Imaging Center, University of Texas Health Science Center in San Antonio, TX) and confirmed with manual inspection using the Talairach atlas (Talaraich and Tournoux, 1988) as a reference.

\section{Results}

Behavioral results

Participants were assessed with the WASI, a brief measure of intelligence. Full-scale IQ scores ranged from 101-141 (mean = 123.7, SD = 12.2). Reaction times (RTs) to videos while in the
Table 1. Scanned participants' postscan ratings of all video segments, using scales from 1 (least) to 8 (most) for funny and enjoyable

\begin{tabular}{lllll}
\hline Stimulus ratings & Funny & Positive & Neutral & Significant differences \\
\hline Mean (SD) & & & & \\
Funny rating & $6.43(1.09)$ & $2.97(1.59)$ & $2.15(1.25)$ & $\mathrm{F}>\mathrm{P}^{*}{ }^{*} \mathrm{~F}>\mathrm{N}^{*}{ }^{*} \mathrm{P}>\mathrm{N}^{*}$ \\
Enjoyable rating & $6.12(1.13)$ & $6.55(1.01)$ & $3.07(1.62)$ & $\mathrm{F}>\mathrm{N}^{*} \mathrm{P}>\mathrm{N}^{*}$ \\
\hline
\end{tabular}

F, Funny; P, Positive; N, Neutral. * $p<0.05$.

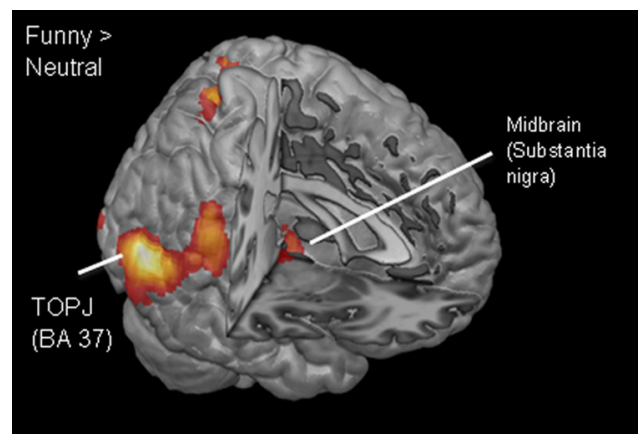

Figure 2. Functional maps demonstrating activations in bilateral temporo-parietaloccipital regions during the Funny $>$ Neutral contrast. All activations shown surpassed a whole-brain voxel-level threshold of $p<0.001$ (uncorrected); those in Table 2 also surpassed cluster-extent threshold of $p<0.05$ (FWE corrected).

scanner were measured for all participants: average (SD) RT in milliseconds for Funny videos $=830.96$ (141.20), Positive $=$ 719.27 (129.68), and Neutral $=911.45$ (155.25). An ANOVA revealed significant $\mathrm{RT}$ differences between trial types $\left(F_{(2)}=\right.$ $8.520, p<0.005)$. Planned contrasts indicated that RTs to Funny videos were significantly slower than to Positive videos $(p<$ $0.005)$, and were significantly faster than RTs to Neutral videos $(p<0.005)$. 
Table 2. fMRI group activation statistics

\begin{tabular}{|c|c|c|c|c|c|c|c|}
\hline \multirow[b]{2}{*}{ BA } & \multirow[b]{2}{*}{ Anatomical location } & \multicolumn{3}{|c|}{ Talairach (peak) coordinate } & \multirow[b]{2}{*}{ Cluster size (voxel) } & \multirow[b]{2}{*}{ zscore } & \multirow[b]{2}{*}{ Peak voxel $p$ value } \\
\hline & & $X$ & $y$ & $z$ & & & \\
\hline \multicolumn{8}{|c|}{ Funny $>$ Neutral } \\
\hline $39 / 40$ & Left temporo-occipital-parietal junction & -44 & -69 & 11 & 3115 & 5.08 & $<0.0005$ \\
\hline \multirow[t]{2}{*}{$37 / 40 / 18$} & Right temporo-occipital-parietal junction & 44 & -60 & 7 & 3100 & 4.98 & $<0.0005$ \\
\hline & *Right midbrain (including substantia nigra) and thalamus* & 16 & -23 & -2 & 249 & 3.86 & $<0.0005$ \\
\hline \multicolumn{8}{|c|}{ Funny $>$ Positive } \\
\hline $39 / 40$ & Left superior temporal gyrus and supramarginal gyrus & -51 & -54 & 12 & 1865 & 5.34 & $<0.0005$ \\
\hline $22 / 40$ & Right superior temporal gyrus and supramarginal gyrus & 50 & -42 & 13 & 1464 & 4.64 & $<0.0005$ \\
\hline \multicolumn{8}{|c|}{ Positive $>$ Neutral } \\
\hline $39 / 19 / 20$ & Right inferior and middle temporal gyrus & 50 & -58 & 7 & 887 & 4.80 & $<0.0005$ \\
\hline $37 / 19$ & Left temporo-occipital-parietal junction & -48 & -64 & 5 & 967 & 4.31 & $<0.0005$ \\
\hline 40 & Right inferior parietal & 61 & -28 & 27 & 868 & 4.36 & $<0.0005$ \\
\hline 40 & Left inferior parietal & -55 & -33 & 33 & 772 & 4.27 & $<0.0005$ \\
\hline $19 / 7 / 5$ & Left precuneus and postcentral gyrus & 30 & -68 & 38 & 641 & 4.08 & $<0.0005$ \\
\hline
\end{tabular}

Unless otherwise noted, all group analyses are presented with a peak-level threshold of $p<0.001$ uncorrected, with a cluster-level threshold of $p<0.05 \mathrm{FWE}$ corrected. ${ }^{*}$ The midbrain cluster was marginally significant at $p=0.094 \mathrm{FWE}$ corrected.

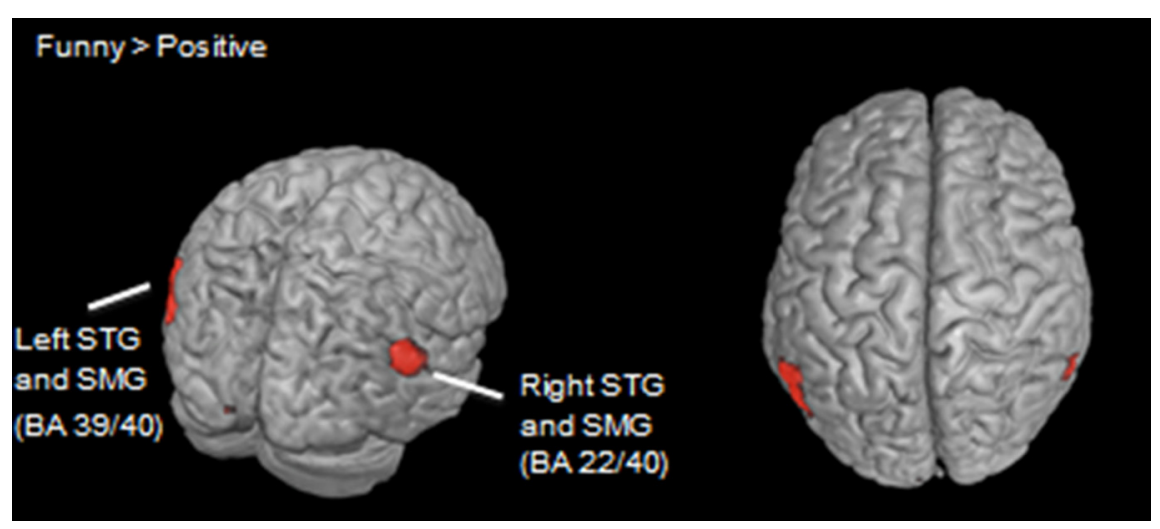

Figure 3. Functional maps demonstrating activations in bilateral temporo-parietal-occipital regions during the Funny $>$ Positive contrast. All activations shown surpassed a whole-brain voxel-level threshold of $p<0.001$ (uncorrected); those in Table 2 also surpassed cluster-extent threshold of $p<0.05$ (FWE corrected). STG, superior temporal gyri; SMG, supramarginal gyri.

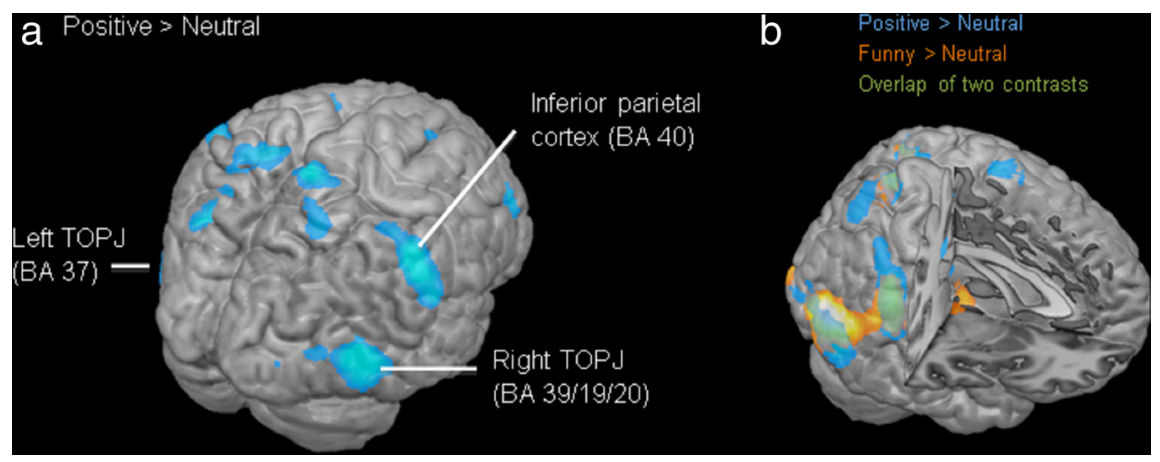

Figure 4. $\quad \boldsymbol{a}$, Functional maps demonstrating activations in bilateral temporo-parietal-occipital regions during the Positive $>$ Neutral contrast. $\boldsymbol{b}$, A comparison of the activations observed for Positive $>$ Neutral videos (blue) and Funny $>$ Neutral (orange/ yellow/white). The overlap of these two contrasts is shown in green. All activations shown surpassed a whole-brain voxel-level threshold of $p<0.001$ (uncorrected); those in Table 2 also surpassed cluster-extent threshold of $p<0.05$ (FWE corrected).

After scanning, all participants evaluated the stimuli on an ordinal scale to provide feedback on how subjectively funny (from $1=$ least to $8=$ most) and enjoyable (from $1=$ least to 8 = most) they found the stimuli. While age-matched participants had previously rated the videos, these postscan ratings from our current participants provide evidence that the stimuli selected were perceived as expected. Funny stimuli were rated by scanned participants as having a mean funny rating of
6.43 and a mean enjoyable rating of 6.12. Positive videos received a mean funny rating of 2.97 and a mean enjoyable rating of 6.55. Neutral videos averaged a funny rating of 2.15 and an enjoyable rating of 3.07 (see Fig. 6, Table 1).

To assess whether age affected participants' postscan ratings of how funny and enjoyable the videos were, we performed Pearson correlations between age and the postscan ratings. We observed a marginally significant negative correlation between age and enjoyable ratings for Funny videos $(r=$ $-0.508, n=15, p=0.053$ ). In addition, we observed a negative relationship between age and postscan ratings of humorousness of Positive stimuli $(r=-0.528, n=15, p<$ $0.05)$. No other correlations reached or approached significance.

\section{fMRI results}

Whole-brain voxel-based functional activation analysis revealed a number of cortical and subcortical structures involved in the detection and appreciation of humor. Unless otherwise noted, all imaging analyses are reported using a joint-expected probability threshold (Poline et al., 1997) with height (peak-level) threshold of $p<0.001$ (uncorrected) and extent (cluster-level) threshold of $p<0.05$ (FWE corrected). In a comparison of the neural response to Funny clips $>$ Neutral clips, we found significantly greater BOLD activation in the left and right TOPJ, extending into the middle temporal gyrus (BA 37). Additional clusters were observed in the left and right occipital lobes (BA 17 and 18) as well as the right inferior parietal lobule (BA 40). In addition, we observed activation in the right midbrain (Tal coordinate peak: $x=16, y=-23, z=-2$; Fig. 2, Table 2).

A comparison of neural activation associated with viewing Funny $>$ Positive videos revealed significant BOLD signal in the left and right superior temporal gyri and supramarginal gyri (BA 40, extending 
to BA 39). These activations were within the TOPJ [which includes BA 39 and 40, along with BA 37 (Luria, 1970)]. A region of middle temporal gyrus further anterior and inferior to TOPJ was also observed (Fig. 3, Table 2).

A contrast of neural activity relating to Positive versus Neutral videos resulted in significant clusters of activation in the left TOPJ (BA 37), as well as a right-lateralized cluster in the right inferior and middle temporal gyrus (BA 39/19/20). In addition, bilateral inferior parietal lobe (BA 40) was active for Positive $>$ Neutral videos (Fig. 4, Table 2).

A multiple regression performed with age as a covariate of interest was used to compare the neural activity in response to Funny versus Positive videos. We found that as children grow older, they are less likely to activate a right-lateralized network including the right inferior frontal gyrus (BA 44), postcentral gyrus (BA 2), and lingual gyrus (BA 19). In addition, the younger the child, the greater the activity in the right NAcc and regions of the brainstem, including substantia nigra and red nucleus (Figs. 5, 6; Table 3). For this exploratory age-related analysis, we used a voxelwise threshold of $p<0.001$ uncorrected, with a minimum cluster (extent) size of 30 voxels.

\section{Discussion}

We observed greater activation in bilateral TOPJ, a region important for resolving incongruity, and the midbrain during children's responses to Funny videos than to Neutral videos. These results are consistent with the humor-related activation found in adults (Mobbs et al., 2003) and suggest that an analogous humor-processing network is already present in middle childhood. Additionally, we observed that TOPJ regions were more active in the contrast of Funny versus Positive videos, indicating that portions of the TOPJ are involved in aspects of humor processing and are distinct from the general circuits that process reward unrelated to humor.

When we compared participants' reactions to Funny versus Neutral clips, we observed activation in the right midbrain (including the substantia nigra), a region previously implicated in the perception of reward (Elliott et al., 2000). We did not observe NAcc activation in this contrast, though it was observed prominently in the study of humor processing in adults viewing static, humorous cartoon stimuli (Mobbs et al., 2003). The lack of activation in the present task may stem from our chosen set of video stimuli that unfold over time. The NAcc response tends to be relatively discrete and is tightly locked to an individual's reward response. The precise timing of the funny event(s) featured in the stimuli differed between clips, as did each child's time to detect the humor. Because the funny events within the videos occurred at differing time points, and because each individual's short, peak NAcc response differed depending on his/her time to detect the humor, the NAcc signal for a given video was diluted across participants. In contrast, the TOPJ activation to process the humorous incongruity was relatively spread out across time and was evident in our results despite the stimuli characteristics.

Activation of the TOPJ has been repeatedly implicated in incongruity resolution and the semantic processing of jokes respectively.

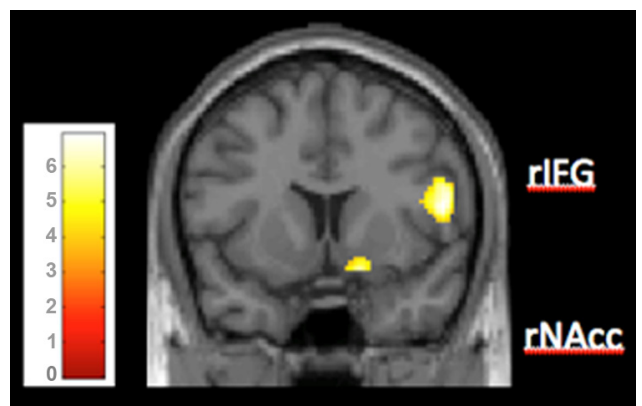

Figure 5. Functional maps demonstrating greater activations in right inferior frontal gyrus (rIFG; BA 44) and right nucleus accumbens (rNAcc) for younger participants in the Funny $>$ Positive contrast. All activations shown surpassed a whole-brain voxel-level threshold of $p<$ 0.001 (uncorrected); those in Table 3 also surpassed cluster-extent threshold $k=30$ voxels.

Figure 6. Postscan mean ratings of clip stimuli. The results of aggregated postscan ratings demonstrate that with few exceptions, clips were assessed in general agreement with the Pilot 1 and Pilot 2 participants' evaluations. Gray symbols represent funny ratings; black symbols resent enjoyable ratings. Funny, Neutral, and Positive clips are represented by squares, circles, and triangles, 
Table 3. Functional activations for Funny $>$ Positive contrast, with age as a covariate of interest

\begin{tabular}{|c|c|c|c|c|c|c|c|}
\hline \multirow[b]{2}{*}{ Location } & \multirow[b]{2}{*}{ Anatomical location } & \multicolumn{3}{|c|}{ Talairach (peak) coordinate } & \multirow[b]{2}{*}{ Cluster size (voxel) } & \multirow[b]{2}{*}{ zscore } & \multirow[b]{2}{*}{ Peak voxel $p$ value } \\
\hline & & $x$ & $y$ & $z$ & & & \\
\hline BA 44 & Right inferior frontal gyrus & 53 & 10 & 12 & 247 & 4.41 & $<0.0005$ \\
\hline BA 2 & Right postcentral gyrus & 57 & -20 & 29 & 119 & 4.06 & $<0.0005$ \\
\hline Putamen & Right nucleus accumbens (putamen) & 16 & 9 & -12 & 168 & 4.01 & $<0.0005$ \\
\hline \multirow[t]{2}{*}{ BA 19} & Right lingual gyrus & 24 & -64 & -2 & 82 & 3.78 & $<0.0005$ \\
\hline & Midline midbrain (substantia nigra and red nucleus) & 14 & -12 & -13 & 313 & 3.76 & $<0.0005$ \\
\hline BA 21 & Right temporal lobe & 40 & -3 & -12 & 78 & 3.49 & $<0.0005$ \\
\hline
\end{tabular}

These activations represent negative correlations—-that is, greater activity for younger participants. Activation maps are presented with a peak-level threshold of $p<0.001$ uncorrected, with a cluster (extent) threshold of 30 voxels.

region of TOPJ reported by Mobbs and colleagues (2003), was not present in this contrast. One interpretation of this finding is that, at least in children, this particular cortical region may not be humor-essential, but is instead associated with rewards, generally. Unexpected rewards, which result in more robust activation of the dopaminergic neurons associated with reward-related neural network (Schultz et al., 2000), also entail incongruity (between expectation and reality).

Though the specific contribution of BA 37 to humor processing is open to interpretation, the contrast of Funny $>$ Positive shows greater activation in more superior TOPJ regions (such as in BA 22, 39, and 40). This indicates that responding to Funny clips employ these TOPJ regions more strongly than to Positive clips. These regions appear to be specific to particular components of humor processing (including the satisfaction of detecting and resolving the incompatible elements of humor, for example) rather than being a component of a more generalized response to reward.

\section{Age-related modulation of humor processing}

Because our participant group spanned a developmental period marked by substantial cognitive and emotional changes (ages 6-12 years old), we wanted to examine age-dependent modulation of neural activity. We performed a multiple regression, using age as a covariate of interest, for the Funny $>$ Positive contrast. We found that the younger the child, the stronger the activation in the NAcc and right inferior frontal gyrus (BA 44; Fig. 5, Table $3)$. The NAcc receives rich dopaminergic input from the ventral striatum (Bates and Goldman-Rakic, 1993) and has long been suggested to play a role in the dopaminergic reward network associated with humor appreciation (Mobbs et al., 2003). Increased activation in this reward-related region in younger participants suggests that younger children find the Funny stimuli more intrinsically rewarding, or are less expectant of its rewarding component, than older children and thus respond more strongly when the reward is present.

It is possible that the increased dopaminergic response found in younger participants is reflective of personal preferences that evolve with age. To optimize appreciation of humor, both the introduced incongruity and the correspondent resolution must present enough of a cognitive challenge to be satisfying. Prior research suggests that jokes just outside of comfortable cognitive mastery are those most preferred across all age groups (Zigler et al., 1966). It is possible that more robust NAcc activation in younger participants' responses reflects age-appropriateness. The Funny clips, largely featuring fairly simplistic violations of expectation, may have been less stimulating for participants at a more advanced stage of development. In addition, though participants showed no differences in their rating of the funniness of Funny videos, younger participants rated the Funny videos as more enjoyable.
Table 4. Qualitative coding of characteristics of interest in videos

\begin{tabular}{llcccl}
\hline & Selected characteristics of interest & Funny & Neutral & Positive & $\begin{array}{l}\text { Significant } \\
\text { difference }\end{array}$ \\
\hline 1 & Number of people (average) & 2.38 & 3.56 & 2.75 & n.s. \\
2 & Number of animals (average) & 0.38 & 0.31 & 0.06 & $\mathrm{~F}>\mathrm{N}^{*}$ \\
3 & Talking during clip & $91 \%$ & $72 \%$ & $41 \%$ & $\mathrm{~F}>\mathrm{P}^{*}$ \\
4 & Location: indoors & $59 \%$ & $53 \%$ & $53 \%$ & n.s. \\
5 & Music & $69 \%$ & $28 \%$ & $69 \%$ & $\mathrm{~F}>\mathrm{N}^{*}$ \\
6 & Laugh-track & $100 \%$ & $0 \%$ & $0 \%$ & $\mathrm{~F}>\mathrm{N}^{*}$ \\
7 & Clip length (s) & 12 & 14 & 13 & n.s. \\
\hline
\end{tabular}

At test was performed to compare characteristics 1,2 , and 7 , while a $\chi^{2}$ test was performed on characteristics 3,4 , 5 , and 6 . While video clip stimuli match fairly well in regards to number of people, indoor/outdoor setting of scenes, and length of clip, there are some marked differences across other characteristics. F, Funny; N, Neutral; P, Positive; n.s., not significant. ${ }^{*} p<0.05$

\section{Stimuli selection}

We coded all video stimuli on a set of quantitative and qualitative dimensions (Table 4). Though we attempted to equate stimuli across as many dimensions as possible, differences between video clip content-including auditory and visual features - may have contributed to variations in activation. For example, a few differences remained between groups, including the universal presence of a laugh-track in Funny clips and its universal absence in Neutral and Positive clips. Interestingly, while Sander and Scheich $(2001,2005)$ found that activation in the amygdala, insula, and auditory regions of the brain is associated with responding to the auditory stimulus of human laughter, none of these regions revealed significant activation in our analyses. This is consistent with the findings of Moran and colleagues (2004), who found equivalent neural activation in participants responding to humorous videos both with and without a laugh track, though it is possible that these findings do not generalize to children.

We also note that differences between video stimuli in our study are likely to be more varied than other featured humorous stimuli. The potential variance in our stimulus set was increased due to the temporal nature of our video stimuli (with hundreds of different frames, each a complex visual image, comprising each video). While canned jokes might allow for more strictly controlled visual variation across stimuli, using such comic media necessitates that participants possess a comparable mastery of language to resolve the incongruities suggested by dual meanings of words or concepts and get the joke. Equivalent cognitive and verbal faculties cannot be assumed in a study examining development in children from 6 to 12 . Video stimuli, however, require less cognitive effort to process, and thus provide a more effective medium through which to engage child participants.

It is possible that the type of humor featured may have influenced activation (Samson et al., 2009). Slapstick comedy in particular does not require the same frontal cortex-powered problem-solving and integration of content requisite of appreciating more nuanced forms of humor (Shammi and Stuss, 1999). 
However, as such, it is an ideal comedic medium for children, whose prefrontal cortices mature through their twenties (Giedd et al., 1999).

In conclusion, our study provides evidence for a humor processing network including the TOPJ and NAcc (similar to the adult network) that is already active in middle childhood. This finding presents a missing link in the longitudinal portrait of humor processing: early cultivation of a neural network devoted to appreciating humor appears to be characteristic of typical development. Still, several components of humor-related neural processing require further study. Future studies should examine the extent to which the response to humorous stimuli is sexdependent in children (as it is in adults), as the ability to process reward (i.e., to perceive something as pleasurable) is impaired in conditions such as major depression-a disorder affecting a disproportionate number of women. Additionally, future work could examine humor's unique contribution to functioning by examining whether neural activation (of both children and adults) differs for humor-related incongruity (as depicted in the present study) versus non-humorous incongruity (e.g., advanced magical illusions). The continued elucidation of the neural correlates and brain basis of humor, and their relationship to the life course, may yield critical insight into how we perceive our relationships, our social world, and ultimately ourselves.

\section{References}

Bates JF, Goldman-Rakic PS (1993) Prefrontal connections of medial motor areas in the rhesus monkey. J Comp Neurol 336:211-228.

Bruno RM, Johnson JM, Simon J (1987) Perception of humor by regular class students and students with learning disabilities or mild mental retardation. J Learn Disabil 20:568-570.

Elliott R, Friston KJ, Dolan RJ (2000) Dissociable neural responses in human reward systems. J Neurosci 20:6159-6165.

Friston KJ, Holmes AP, Poline JB, Grasby PJ, Williams SC, Frackowiak RS, Turner R. (1995) Analysis of fMRI time-series revisited. Neuroimage 2:45-53.

Giedd JN, Blumenthal J, Jeffries NO, Castellanos FX, Liu H, Zijdenbos A, Tomáš P, Evans AC, Rapoport JL (1999) Brain development during childhood and adolescence: a longitudinal MRI study. Nat Neurosci 2:861863.

Glover GH, Lai S (1998) Self-navigated spiral fMRI: interleaved versus single-shot. Magn Reson Med 39:361-368.

Goel V, Dolan RJ (2001) The functional anatomy of humor: segregating cognitive and affective components. Nat Neurosci 4:237-238.
Johnson K, Mervis CB (1997) First steps in the emergence of verbal humor: a case study. Infant Behav Dev 20:187-196.

Kim DH, Adalsteinsson E, Glover GH, Spielman DM (2002) Regularized higher-order in vivo shimming. Magn Reson Med 48:715-722.

Luria, AR (1970) Traumatic aphasia: its syndromes, psychology, and treatment. The Netherlands: Mouton.

Martin RA (2007) The psychology of humor: an integrative approach (1st ed). Burlington, MA: Academic.

Mobbs D, Greicius MD, Abdel-Azim E, Menon V, Reiss AL (2003) Humor modulates the mesolimbic reward centers. Neuron 40:1041-1048.

Moran JM, Wig GS, Adams RB Jr, Janata P, Kelley WM (2004) Neural correlates of humor detection and appreciation. Neuroimage 21:1055-1060.

Pinderhughes EE, Zigler E (1985) Cognitive and motivational determinants of children's humor responses. J Res Person 19:185-196.

Poline JB, Worsley KJ, Evans AC, Friston KJ (1997) Combining spatial extent and peak intensity to test for activations in functional imaging. Neuroimage 5:83-96.

Samson AC, Hempelmann CF, Huber O, Zysset S (2009) Neural substrates of incongruity-resolution and nonsense humor. Neuropsychologia 47:1023-1033.

Sander K, Scheich H (2001) Auditory perception of laughing and crying activates human amygdala regardless of attentional state. Brain Res Cogn Brain Res 12:181-198.

Sander K, Scheich H (2005) Left auditory cortex and amygdala, but right insula dominance for human laughing and crying. J Cogn Neurosci 17:1519-1531.

Schultz W (2002) Getting formal with dopamine and reward. Neuron $36: 241-263$

Schultz W, Tremblay L, Hollerman JR (2000) Reward processing in primate orbitofrontal cortex and basal ganglia. Cereb Cortex 10:272-284.

Shammi P, Stuss DT (1999) Humour appreciation: a role of the right frontal lobe. Brain 122:657-666.

St. James PJ, Tager-Flusberg H (1994) An observational study of humor in autism and down syndrome. J Autism Dev Disord 24:603-617.

Suls JM (1972) A two-stage model for the appreciation of jokes and cartoons. In: Psychology of Humor (Goldstein JH, McGhee PE, eds.). NY: Academic.

Szaflarski JP, Holland SK, Schmithorst VJ, Byars AW (2006) fMRI study of language lateralization in children and adults. Hum Brain Mapp 27:202-212

Talaraich J and Tournoux P (1988) Co-planar stereotaxic atlas of the human brain: 3-dimensional proportional system: an approach to cerebral imaging. New York: Thieme Medical Publishers.

Yamada Y, Stevens C, Dow M, Harn B, Chard D, Neville H (2011) Emergence of the neural network for reading in five-year-old beginning readers of different levels of pre-literacy abilities: an fMRI study. Neuroimage 57:704-713.

Zigler E, Levine J, Gould L (1966) Cognitive processes in the development of children's appreciation of humor. Child Dev 37:507-518. 